\title{
Superficies Acotadas y Sólidos no Convencionales en GeoGebra
}

\author{
Alejandro I. Flores Osorio', Clara R. Moncada Andino \\ ${ }^{1}$ Universidad Peruana de Ciencias Aplicadas - Departamento de Ciencias Básicas \\ Código Postal 15067 - Lima - Perú \\ ${ }^{2}$ Instituto GeoGebra de Zacatepec - Departamento de Ciencias Básicas \\ Tecnológico Nacional de México/Campus Zacatepec \\ Código Postal 62780 - Morelos - México \\ alexflores277@gmail.com, clara.moncada@gmail.com
}

\begin{abstract}
This research involves the use of GeoGebra to establish in an interactive and dynamic way the graph of bounded surfaces and unconventional solids, thus improving students' understanding, abstraction and visualization of the course of calculation of several variables. In this course it is necessary to represent these graphs, to determine their volume, mass or center of mass using multiple integrals. These surfaces can be defined by means of a parameterization based on two variables to be inserted in the GeoGebra 3D graphic interface using the Surface command. This work focuses on graphing using cylindrical coordinates for some bounded surfaces and unconventional solids.
\end{abstract}

Resumen. Esta investigación involucra el uso de GeoGebra para establecer en forma interactiva y dinámica la gráfica de superficies acotadas y sólidos no convencionales, de esta manera mejorar la comprensión, abstracción y visualización de los estudiantes del curso de cálculo de varias variables. En este curso es necesario representar estas gráficas, para determinar su volumen, masa o centro de masa usando integrales múltiples. Estas superficies pueden definirse mediante una parametrización en función de dos variables a insertar en la interfaz gráfica 3D de GeoGebra mediante el comando Superficie. Este trabajo se centra en graficar usando las coordenadas cilindricas para algunas superficies acotadas y sólidos no convencionales.

\section{Introducción}

En el estudio del cálculo de varias variables es necesario establecer el esbozo de superficies en $\mathrm{R}^{3}$ o de sólidos que son acotados por un conjunto finito de superficies, representar la gráfica de estas superficies sobre una región plana como es el caso de una pizarra o una simple hoja de papel representa para los estudiantes una gran dificultad ya que la gráficas de muchas de estas superficies y de sólidos acotados por estas superficies son desconocidas, la dificultad se acrecienta ya que esto conlleva a tener una buena perspectiva y abstracción de dichas superficies sobre el plano.

Es ante esta dificultad que resulta útil trabajar con un software matemático que permita un manejo sencillo, una interfaz gráfica dinámica e interactiva que pueda plasmar la gráfica de las superficies y de sólidos generados por éstas. GeoGebra permite plasmar en forma dinámica e interactiva la gráfica de elementos en el espacio tridimensional en su 
vista gráfica 3D. El esbozo de las gráficas de las superficies ingresadas directamente desde la barra de entrada del GeoGebra nos permite visualizar la gráfica de superficies no acotadas; aunque la interfaz gráfica de GeoGebra permite visualizar en diferentes ángulos las superficies y los sólidos que se generan por la intersección de estas superficies; es necesario establecer la construcción del gráfico de las superficies acotadas. Es en este punto donde es importante parametrizar las superficies y de acuerdo a los resultados que se obtengan, expresarlos matemáticamente mediante el comando Superficie del GeoGebra.

La interfaz gráfica 3D del GeoGebra crea un ambiente de trabajo en el cual se pueden visualizar en forma dinámica e interactiva las trazas de una superficie, curvas de intersección entre superficies, proyecciones de una superficie sobre un plano coordenado. El comando Superficie del GeoGebra permite trabajar con parametrizaciones y con ello se puedes esbozar superficies parametrizadas o superficies acotadas. En consecuencia los ambientes enriquecidos con TIC proporcionan un escenario flexible y adecuado para el aprendizaje; eliminando las barreras del espacio y el tiempo en la interacción entre el docente y los estudiantes, incrementando la comunicación y el aprendizaje autónomo en el estudiante [Massa, 2015].

\section{Fundamentos Matemáticos}

\subsection{Coordenadas Cilíndricas}

Para representar gráficamente objetos en el espacio se utilizan sistemas coordenados, entre ellos: de coordenadas cartesianas, cilíndricas, esféricas, etc. Este trabajo se enfoca en el sistema de coordenadas cilíndricas que son resultado de la generalización del sistema de coordenadas polares en el plano, siendo uno de sus usos la solución de problemas con simetría axial. Considerando que en el sistema de coordenadas cilíndricas, un punto $P(x, y, z)$ en el espacio es representado por la terna ordenada $(r, \theta, z)$, donde $(r, \theta)$ es una representación en coordenadas polares de la proyección del punto en el plano coordenado $X Y$ y $Z$ es la distancia dirigida de $(r, \theta)$ al punto $P$, como se muestra en la figura 1, a partir de los enfoques que Larson (2010) y Stewart (2012) hacen.

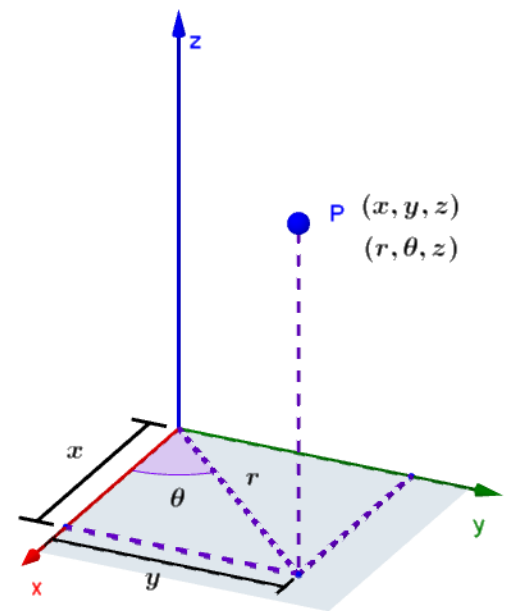

Figura 1. Coordenadas del punto $P$ 
VIII Congresso Brasileiro de Informática na Educação (CBIE 2019)

Anais dos Workshops do VIII Congresso Brasileiro de Informática na Educação (WCBIE 2019)

Para la conversión de coordenadas, de rectangulares a cilíndricas y viceversa, se usa la siguiente relación:

De cilíndricas a rectangulares:

$$
\left\{\begin{array}{l}
x=r \cos \theta \\
y=r \operatorname{sen} \theta, 0 \leq \theta \leq 2 \pi \wedge r>0 \\
z=z
\end{array}\right.
$$

De rectangulares a cilíndricas:

$$
\left\{\begin{array}{c}
r^{2}=x^{2}+y^{2} \\
\tan \theta, \quad x \neq 0 \\
z=z
\end{array}\right.
$$

\subsection{Superficies Paramétricas}

Sean las funciones $x, y$ y $z$ de variables $u$ y $v$, continuas en un dominio $D$ del plano $u v$. Se denomina superficie paramétrica al conjunto de puntos $(x, y, z)$ dado por

$$
\mathbf{r}(u, v)=x(u, v) \mathbf{i}+y(u, v) \mathbf{j}+z(u, v) \mathbf{k}
$$

Donde las ecuaciones

$$
\left\{\begin{array}{l}
x=x(u, v) \\
y=y(u, v) \\
z=z(u, v)
\end{array}\right.
$$

Son las ecuaciones paramétricas de la superficie.

\subsection{Graficando Superficies}

Usando coordenadas cilíndricas y la definición de superficies paramétricas se va a graficar superficies acotadas en GeoGebra, para tal efecto se va a utilizar el comando Superficie del GeoGebra, cuya sintaxis está establecida de la siguiente forma:

Superficie (<Expresión $>,<$ Expresión $>,<$ Expresión $>,<$ Parámetro1 $>,<$ Valor inicial1 $>,<$ Valor final1 $>,<$ Parámetro2 $>,<$ Valor inicial2>,<Valor final2>)

De acuerdo a la sintaxis establecida del comando superficie, esbozar la gráfica de las siguientes superficies: $S_{1}: z=\sqrt{x^{2}+y^{2}}$ y $S_{2}: z=6-x^{2}-y^{2}$. La gráfica de ambas superficies acotadas hasta la curva donde se intersectan sus superficies va a generar el esbozo de la gráfica de un sólido que es muy diferente a las figuras tridimensionales conocidas, a estos sólidos se les denomina sólidos no convencionales.

Considerando el cambio de coordenadas cartesianas a cilíndricas, definido por:

$$
\begin{gathered}
x^{2}+y^{2}=r^{2} \\
\left\{\begin{array}{l}
x=r \cos t \\
y=r \operatorname{sen} t
\end{array}\right.
\end{gathered}
$$

Se realizan las siguientes consideraciones de equivalencia: 


$$
\begin{array}{rc}
S_{1}: z=\sqrt{x^{2}+y^{2}} & S_{2}: z=6-x^{2}-y^{2} \\
S_{1}: z^{2}=x^{2}+y^{2} & S_{2}: z=6-\left(x^{2}+y^{2}\right) \\
S_{1}: z^{2}=r & S_{2}: z=6-r^{2}
\end{array}
$$

Al igualar ambas ecuaciones $z=6-z^{2}$, se obtiene que la igualdad se cumple para los valores de $z=-3 \vee z=2$, como se ha establecido $S_{1}$ es un semicono que se encuentra por encima del plano $z=0$, de acuerdo a esto se va a considerar el valor de $z=2$, por tanto también se tiene que $r=2$. La ecuación de la curva de intersección de ambas superficies es representada de la siguiente forma:

$$
C:\left\{\begin{array}{c}
x^{2}+y^{2}=4 \\
z=2
\end{array}\right.
$$

El esbozo de la gráfica de las superficies y de la curva de intersección se muestra en la figura 2.

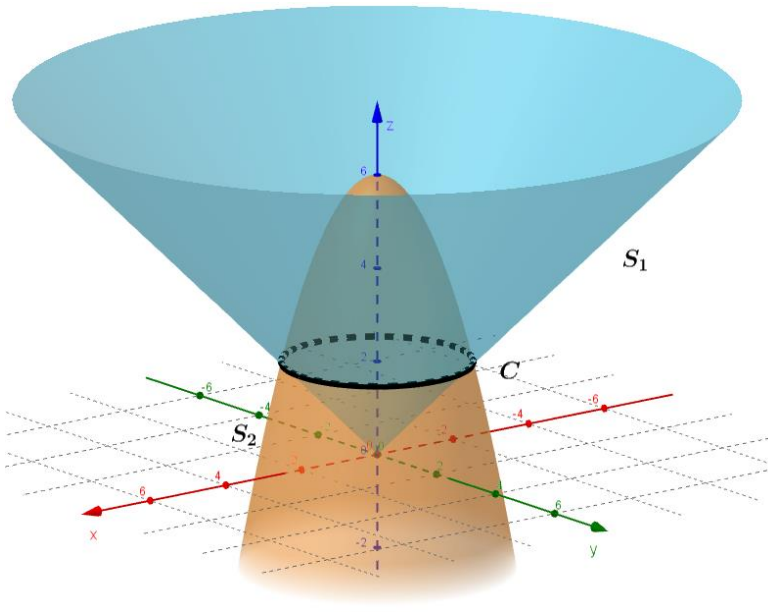

Figura 2. Gráfica de la curva $C$.

De acuerdo a lo obtenido se hace la parametrización la superficie $S_{1}$, usando coordenadas cilíndricas y acotándola de la siguiente manera:

$$
S_{1}:\left\{\begin{array}{c}
x=r \cos t \\
y=r \operatorname{sen} t, \quad t \in[0,2 \pi] \wedge r \in[0,2] \\
z=r
\end{array}\right.
$$

Usando el comando Superficie de GeoGebra con la parametrización establecida, se tiene la sintaxis siguiente, para la Superficie $\mathrm{S}_{1}$ :

$$
\text { Superficie }(r \cos (t), r \operatorname{sen}(t), r, r, 0,2, t, 0,2 \pi)
$$

Que permite visualizar el gráfico de la superficie $S_{1}$ como en la figura 3 . 
VIII Congresso Brasileiro de Informática na Educação (CBIE 2019)

Anais dos Workshops do VIII Congresso Brasileiro de Informática na Educação (WCBIE 2019)

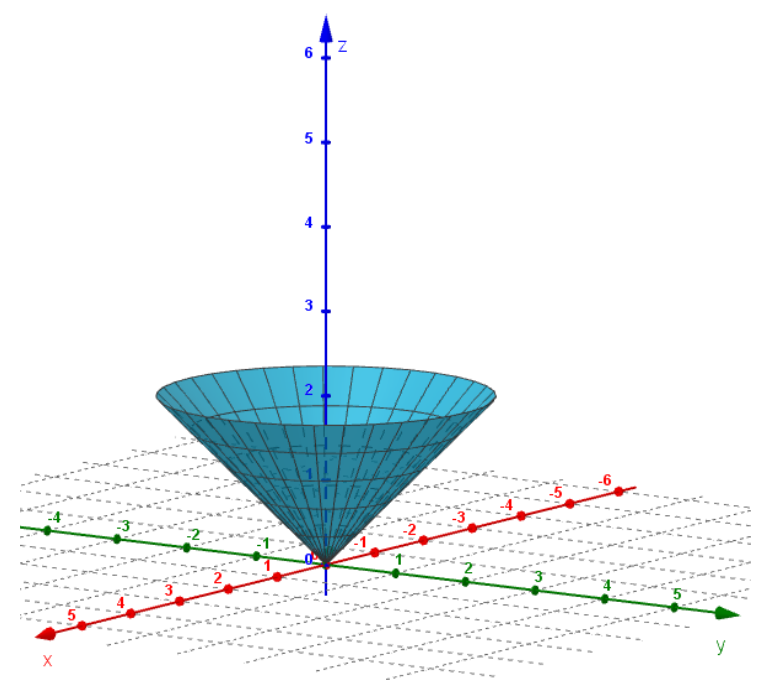

Figura 3. Curva de la superficie $S_{1}$

En el caso de la superficie $S_{2}$, definida por:

$$
S_{2}:\left\{\begin{array}{l}
x=r \cos t \\
y=r \operatorname{sen} t \\
z=6-r^{2}
\end{array} \quad t \in[0,2 \pi] \wedge r \in[0,2]\right.
$$

Se usa el comando Superficie de GeoGebra con la parametrización establecida y se tiene la sintaxis siguiente, para la Superficie $\mathrm{S}_{2}$ :

$$
\text { Superficie }(r \cos (t), r \operatorname{sen}(t), 6-r 2, r, 0,2, t, 0,2 \pi)
$$

Se observa en la figura 4 el esbozo del gráfico de la superficie $S_{2}$.

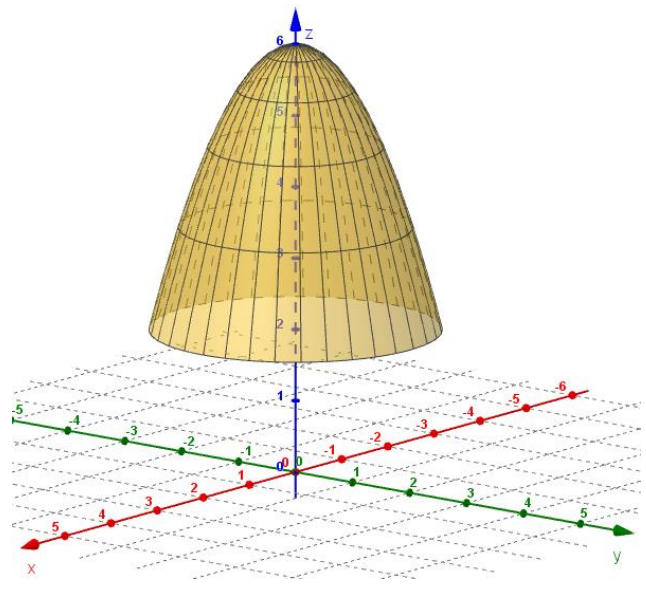

Figura 4. Curva de la superficie $S_{2}$

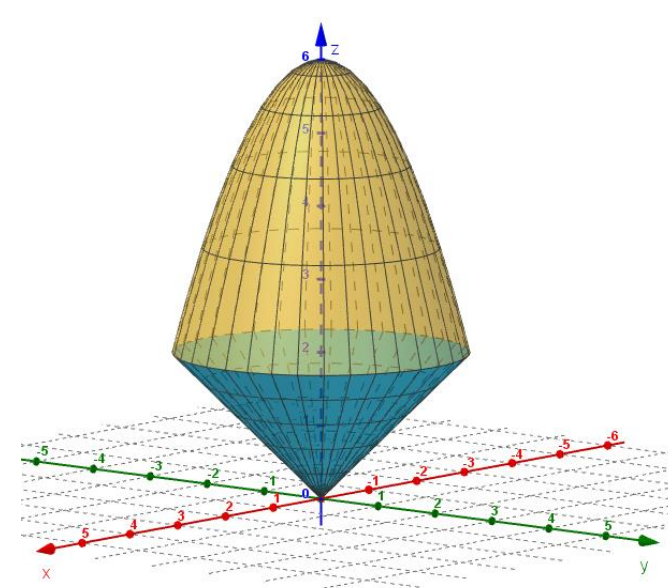

Figura 5. Sólido no convencional

Plasmando ambas superficies parametrizadas y acotados; se visualiza en la figura 5 el sólido no convencional que se genera. 
VIII Congresso Brasileiro de Informática na Educação (CBIE 2019)

Anais dos Workshops do VIII Congresso Brasileiro de Informática na Educação (WCBIE 2019)

Suponiendo que se desea esbozar la superficie representada por la siguiente ecuación $z=6-3 x^{2}-2 y^{2}$, es sumamente sencillo establecer el grafico de la superficie en forma directa en GeoGebra como se observa en la figura 6.

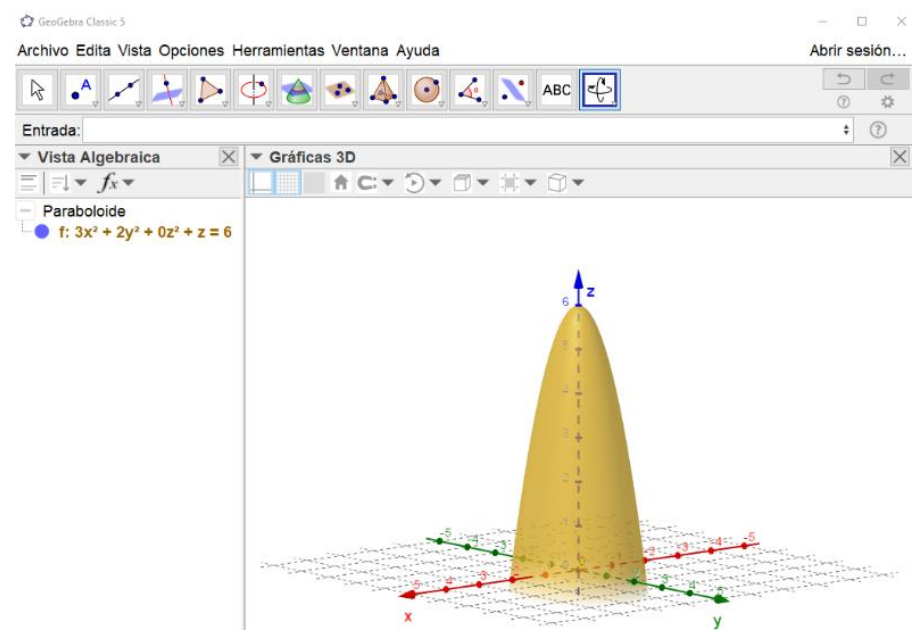

Figura 6. Superficie de $z=6-3 x^{2}-2 y^{2}$

Si se desea esbozar la gráfica de la superficie acotada para $z$ entre 0 y 6 , se hacer uso de la parametrización trigonométrica y de secciones planas que se construyen paralelas al plano $x y$. Para ello, de la ecuación $z=6-3 x^{2}-2 y^{2}$, se buscan secciones para cualquier altura $k$, por lo tanto, con $z=k$, se obtiene:

$$
\begin{aligned}
& \Rightarrow k=6-3 x^{2}-2 y^{2} \\
& \Rightarrow 6-k=3 x^{2}+2 y^{2} \\
& \Rightarrow 1=\frac{3 x^{2}}{6-k}+\frac{2 y^{2}}{6-k}
\end{aligned}
$$

Considerando la ecuación identidad:

$$
1=\cos ^{2}(t)+\operatorname{sen}^{2}(t)
$$

Al sustituir y despejar se obtiene que:

$$
\begin{aligned}
& \cos ^{2}(t)=\frac{3 x^{2}}{6-k} \\
& \operatorname{sen}^{2}(t)=\frac{2 y^{2}}{6-k}
\end{aligned}
$$

Quedando al despejar y parametrizar:

$$
\left\{\begin{array}{l}
x=\sqrt{\frac{6-k}{3}} \cos t \\
y=\sqrt{\frac{6-k}{3}} \operatorname{sen} t \\
z=k
\end{array}, t \in[0,2 \pi] \wedge k \in[0,6]\right.
$$


VIII Congresso Brasileiro de Informática na Educação (CBIE 2019)

Anais dos Workshops do VIII Congresso Brasileiro de Informática na Educação (WCBIE 2019)

Trasladando con los comandos de GeoGebra, primero se establece un deslizador con las características siguientes, mostradas en la figura 7:

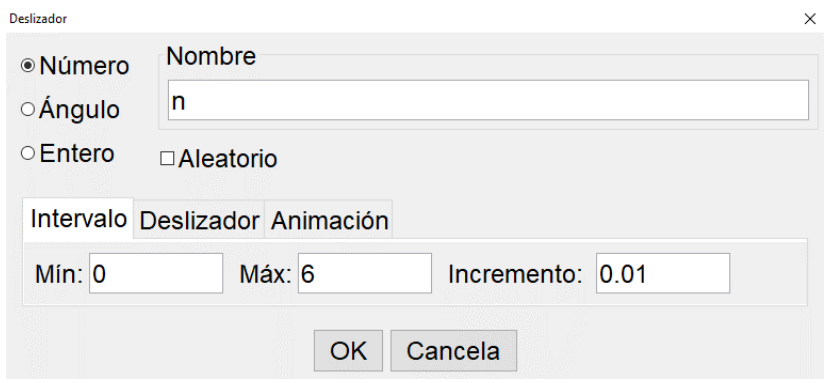

Figura 7. Caracterización de un deslizador

Segundo, se usa el comando Superficie de GeoGebra:

Superficie (<Expresión $>,<$ Expresión $>,<$ Expresión $>,<$ Parámetro1 $>,<$ Valor inicial1 $>,<$ Valor final1 $>,<$ Parámetro2 $>,<$ Valor inicial2 $>,<$ Valor final2 $>$ )

En cada "Expresión" se coloca cada componente de la parametrización:

Superficie $(\operatorname{sqrt}((6-k) / 3 \cos (t), \operatorname{sqrt}((6-k) / 3 \operatorname{sen}(t), k, t, 0,2 \pi, k, 0, n)$

Al momento de manipular el deslizador n, se observa cómo se construye la superficie acotada (ver figura 8).

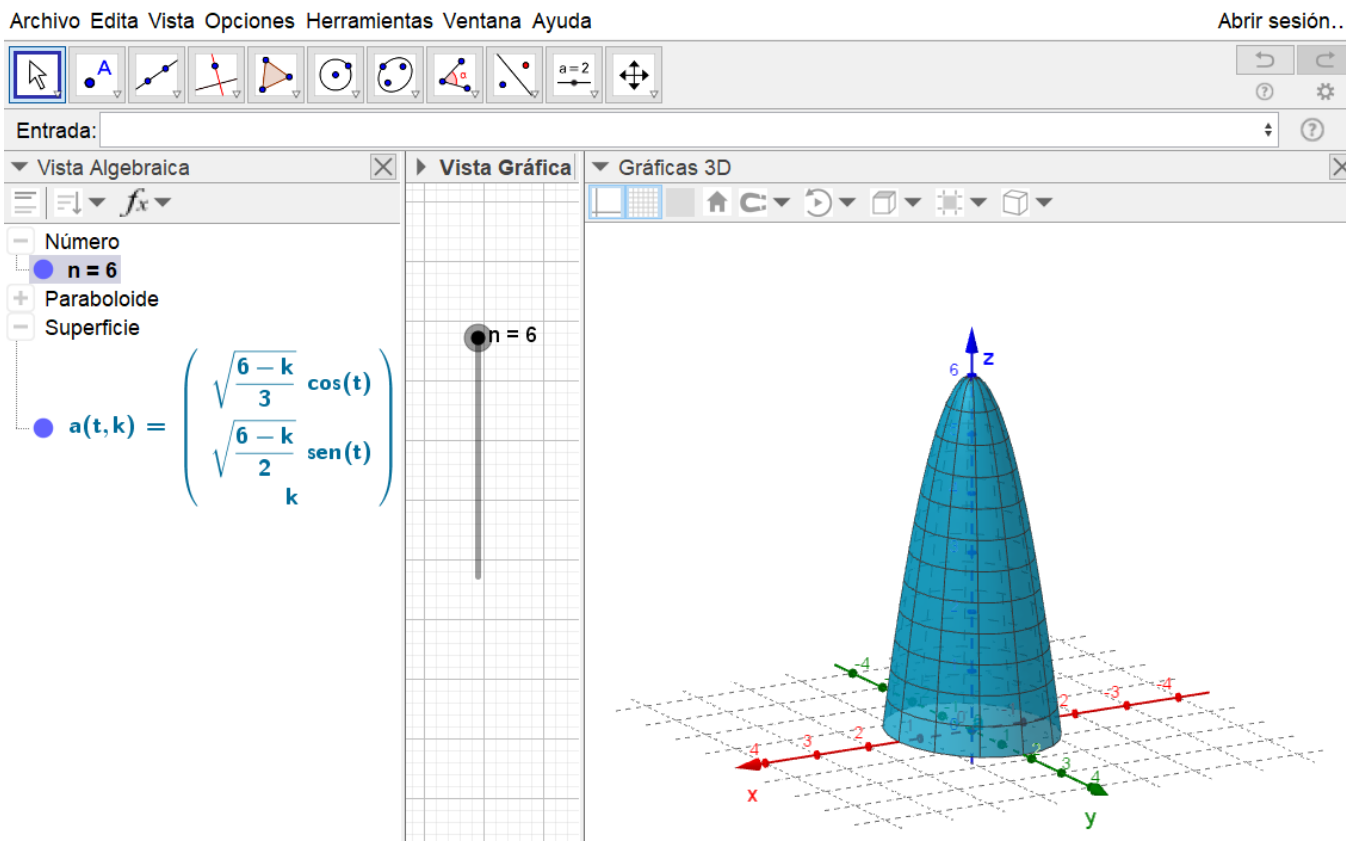

Figura 8. Superficie acotada

Como se ha podido apreciar a lo largo de este trabajo, el recurso virtual tiene su atractivo como herramienta didáctica, al contar con diseño de construcciones interactivas que permiten crear, recrear, simular situaciones en contextos utilizando las herramientas de GeoGebra, que en este trabajo se ha enfocado en el comando Superficie. De esta manera, la posibilidad de interactuar, permite a los estudiantes, de diversas carreras, relacionar y comprobar por sí mismos los conceptos teóricos, lo que les conduce a una 
VIII Congresso Brasileiro de Informática na Educação (CBIE 2019)

Anais dos Workshops do VIII Congresso Brasileiro de Informática na Educação (WCBIE 2019)

mejor comprensión de las aplicaciones de las formas y funciones en la vida real [Cruz y Medina, 2015].

\section{Conclusiones}

El uso de la geometría dinámica permite que el estudiante pueda obtener una comprensión de los conceptos teóricos y plasmar sus aplicaciones en forma interactiva en un entorno visual espacial. Es de esta manera que GeoGebra cumple un rol importante en la construcción de aprendizajes significativos en el estudiante, ya que en forma dinámica e interactiva se accede a la geometría, el álgebra, la estadística y el cálculo. Para esta situación en particular se ha involucrado la interfaz algebraica con la interfaz gráfica 3D de GeoGebra, en el cual se visualizan superficies parametrizadas mediante el uso del comando Superficie, para poder plasmar la gráfica de estas superficies es fundamental establecer una parametrización adecuada de esa superficie de interés y particularizada; es de esta manera que se construyen superficies acotadas, basándose, en esta caso, de la parametrización en coordenadas cilíndricas y con estas superficies se ha construido nuevos sólidos que son acotados y que cuya forma no están catalogadas dentro de los sólidos convencionales, como el cubo o el prisma; a estos sólidos se les ha catalogado como sólidos no convencionales.

La interfaz gráfica de 3D del GeoGebra permite en forma dinámica e interactiva visualizar las superficies de diferentes ángulos, efectuar la rotación sobre un eje específico, proyectar los sólidos no convencionales construidos sobre un plano coordenado y en esta proyección establecer una descripción analítica o en coordenadas cilíndricas y de esta forma calcular el volumen, masa o centro de masa del sólido mediante el uso de integrales múltiples.

Se debe alentar a los estudiantes y docentes a establecer conexiones entre las definiciones y teoremas presentados en el cálculo multivariable y desarrollar nuevas experiencias, formas creativas y actividades de aprendizaje que puedan ser diseñadas en GeoGebra, además de contar con la gratuidad de este software matemático.

\section{Referencias}

Cruz, J., y Medina, Y. (2015) Matemáticas y Realidad: una conexión posible con GeoGebra. Editorial Académica Española, de LAP Lambert Academic Publishing $\mathrm{GmbH} \&$ Co. Alemania.

Massa, S. y Pirro, A. (2015). Formación docente universitaria en competencias para la incorporación de las TICs: dimensiones de análisis. Recuperado de: http://reposital.cuaed.unam.mx:8080/jspui/bitstream/123456789/4148/1/VE14.315.p df.

Larson, R. y Edwards, B. (2010). Cálculo 2 de varias variables (9na Edición). México, D.F. McGraw Hill.

Stewart, J. (2012). Cálculo de varias variables trascendentes tempranas (7ma Edición). México, D.F. Cengage Learning Editores. 